\title{
Gamma-rays from the pulsar wind nebulae
}

\author{
W. Bednarek and M. Bartosik \\ Department of Experimental Physics, University of Łódź, ul. Pomorska 149/153, 90-236 Łódź, Poland \\ Received 13 February 2003 / Accepted 14 April 2003

\begin{abstract}
We investigate the radiation processes inside supernova remnants which are powered by young pulsars. Using a recent model for particle acceleration by the pulsar wind nebulae (PWNe), in which positrons gain energy in the process of resonant scattering by the heavy nuclei, we construct a time-dependent radiation model for the PWNe. In this model, the spectra of relativistic particles, injected inside the nebula, depend on time due to the evolution of the pulsar parameters. Applying a simple model for the evolution of the PWNa, the equilibrium spectra of leptons and nuclei inside the nebula are determined as a function of time, taking into account the energy losses of particles on different processes and their escape from the nebula. We calculate the multiwavelength photon spectra produced by leptons and nuclei and compare them with the observations of the PWNe for which $\mathrm{TeV} \gamma$-ray emission has been reported, i.e. the Crab Nebula, the Vela Supernova Remnant, and the nebula around PSR 1706-44. It is found that the emission from the Crab Nebula can be well fitted by the composition of the $\gamma$-ray emission produced by leptons (below $\sim 10 \mathrm{TeV}$ ) and nuclei $(\sim 10 \mathrm{TeV})$. The model is further tested by successful fitting of the high energy spectrum from the Vela SNR. In this case, the observed $\gamma$-ray emission is mainly due to leptons and the contribution of $\gamma$-rays from decay of neutral pions, produced in collision of nuclei, is significantly lower. However, the considered model does not give good fitting to the emission from PSR1706-44 for the likely parameters of this source, unless an additional target for relativistic leptons is present inside the nebula, e.g. the thermal infrared emission. Based on the knowledge obtained from these fittings, we predict the $\gamma$-ray fluxes in the TeV energy range from other PWNe, which are promissing $\mathrm{TeV} \gamma$-ray sources due to their similarities to the $\gamma$-ray nebulae, i.e. MSH15-52 (PSR 1509-58), 3C58 (PSR J0205+6449), and CTB80 (PSR 1951+32). Possible detection of these sources by the new generation of Cherenkov telescopes is discussed.
\end{abstract}

Key words. ISM: supernova remnants - stars: pulsars: general - gamma-rays: theory - radiation mechanisms: non-thermal

\section{Introduction}

Young pulsars, born in the supernova explosions, create relativistic winds which in the early stage of evolution interact with the supernova ejecta creating the pulsar wind nebulae. These nebulae are filled with energetic particles which can radiate in all range of electromagnetic spectrum. Up to now, several objects of this type have been observed (e.g. Chevalier 2003). Three nebulae, the Crab Nebula and the nebulae around the Vela and PSR1706-44 pulsars, have been detected in $\mathrm{TeV} \gamma$-rays (see for review e.g. Fegan 2001). The best studied object, the Crab Nebula, shows $\gamma$-ray emission up to $50 \mathrm{TeV}$ with a very well-established spectrum, determined by several independent telescopes starting from the original detection by the Whipple group (Weekes et al. 1989). Recently, another PWNa, MSH15-52 around the pulsar PSR 1509-58, has been marginally detected by the CANGAROO telescope (Sako et al. 2000).

It is widely argued that lower energy radiation in the PWNa is produced by leptons in the magnetic field (the synchrotron radiation) and the higher energy part of the spectrum is produced by leptons in the inverse Compton scattering of the low energy synchrotron, Microwave Background

Send offprint requests to: $\mathrm{W}$. Bednarek, e-mail: bednar@fizwe4.fic.uni.lodz.pl
Radiation (MBR), or infrared photons. Leptons are accelerated as a result of interaction of the pulsar wind with the nebula (e.g. Kennel \& Coroniti 1984). First discussions of these radiation processes, with the application to the Crab Nebula, were given by Gould (1965) and Rieke \& Weekes (1969) and a more physical models were analyzed by Grindlay \& Hoffman (1971) and Stepanian (1980). More recently, detailed modeling of the observed high energy emission from the Crab Nebula have been presented by e.g., de Jager \& Harding (1992), Atoyan \& Aharonian (1996), de Jager et al. (1996), and Hillas et al. (1998). Also high energy processes in other PWNe have been discussed in more detail, concentrating on the sources from which detection of $\gamma$-ray photons have been claimed, e.g. nebulae around: PSR 1706-44 (Aharonian et al. 1997), PSR 1509-58 (Du Plessis et al. 1995), Vela pulsar (De Jager et al. 1996b). For example, Aharonian et al. (1997) consider in detail the case of nebula around PSR 1706-44, assuming that leptons are injected continuously during the lifetime of the pulsar with the constant rate and the power law spectrum.

It is likely that also production of $\gamma$-rays in the interactions of hadrons with the matter of the supernova can contribute to the observed spectrum in its higher energy end, especially in the case of younger nebulae (see e.g. Cheng et al. 1990; Aharonian \& Atoyan 1996; Bednarek \& Protheore 1997). 
In fact, the presence of heavy nuclei inside PWNe is quite likely since the significant part of the energy lost by the pulsar can be taken by relativistic iron nuclei. These nuclei excite waves, just after the pulsar wind shock, which energy can be resonantly transfered to positrons (Hoshino et al. 1992). Apart from providing efficient mechanism for acceleration of leptons inside the PWNe, such model can also successfully explain the appearance of thin synchrotron filaments in the Crab Nebula (Gallant \& Arons 1994).

Based on the recent works by Arons and collaborators (see e.g. Arons 1998), we constrain the time-dependent model for the acceleration, propagation, and radiation of particles inside the PWNe. It is assumed that pulsar injects into the nebula relativistic heavy nuclei, with the Lorentz factors determined by the pulsar parameters, which evolve in time due to the pulsar rotational energy losses (see e.g. Gallant \& Arons 1994). Therefore, the injection spectra of leptons and nuclei are time dependent as well. Leptons and nuclei are injected into the surrounding nebula, which parameters also evolve in time, due to expansion of the nebula. The parameters of the nebula are in turn determined by the initial kinetic energy of the supernova, the energy supply by the pulsar, and the surrounding medium. We calculate the equilibrium spectra of leptons and nuclei inside the nebula as a function of time, taking into account radiation and adiabatic energy losses and escape of particles from the nebula. Then, we calculate the photon spectra produced by these particles in processes, which are likely to contribute to the multiwavelength emission from the PWNe.

The paper is organized as follows. We constrain the timedependent model for the expending nebula in Sect. 2, following the early ideas developed by Ostriker \& Gunn (1971) and Rees \& Gunn (1974). In Sect. 3 the model for the acceleration of heavy ions and leptons inside the nebula is described. In Sects. 4 and 5, the equilibrium spectra of particles inside nebula are obtained and the radiation mechanisms are discussed. Finally, in Sect. 6, we confront such a model with the observations of the Crab Nebula, the Vela nebula, and the nebula around PSR 1706-44. From the comparison of calculated spectra with the observations of these nebulae, we derive some free parameters of the considered model in order to predict the level of $\gamma$-ray emission for other nebulae: 3C58 around PSR J0205+6449, CTB80 around PSR 1951+32, and MSH 15-52 around PSR 1509-58.

\section{Interaction of the pulsar with the supernova envelope}

The rotational energy of pulsars, formed during supernova explosion, can be comparable (or even higher) to the kinetic energy of expending envelopes. This energy is lost by the pulsar in the form of relativistic particles and electromagnetic waves, whose energy is absorbed by the envelope. Therefore, in the case of very young pulsars, the parameters of expending envelopes are determined by not only the initial parameters of the envelope and the surrounding medium but also by the energy loss rate of the pulsars.
Since in this paper we intend to consider the case of supernova exploding with arbitrary initial parameters for the neutron star and the envelope, the transfer of energy from the pulsar to the envelope has to be taken into account. In fact, the evolution of the supernova envelope under the influence of the pulsar has been considered soon after the pulsar discovery by Ostriker \& Gunn (1971) (see also later works by e.g. Pacini \& Salvati 1973 or Reynolds \& Chevalier 1984). In order to determine the parameters of the envelope surrounding the pulsar, we follow general prescription presented in this first paper. Let us assume that at the moment of explosion the expansion velocity of the supernova envelope at its inner radius is $V_{0, \mathrm{Neb}}$ and its initial mass is $M_{0, \mathrm{Neb}}$. The expansion velocity, $v_{\mathrm{Neb}}(t)$, increases at the early stage, due to the additional supply of energy to the nebula by the pulsar, and decreases at the later stage, due to the accumulation of the surrounding matter. At an arbitrary time, $t$, it can be determined by analyzing the energy budget of the envelope by applying the simple equation,

$$
\frac{M_{\mathrm{Neb}}(t) V_{\mathrm{Neb}}^{2}(t)}{2}=\frac{M_{0, \mathrm{Neb}} V_{0, \mathrm{Neb}}^{2}}{2}+\int_{0}^{t} L_{\mathrm{pul}}\left(t^{\prime}\right) \mathrm{d} t^{\prime}
$$

where

$L_{\mathrm{pul}}(t)=B_{\mathrm{s}}^{2} R_{\mathrm{s}}^{6} \Omega^{4} / 6 c^{3} \approx 3 \times 10^{43} B_{12}^{2} P_{\mathrm{ms}}^{-4} \operatorname{erg~s}^{-1}$,

is the rate of pulsar energy lost on emission of the dipole electromagnetic radiation, $\Omega=2 \pi / P$, and the period of the pulsar $P=10^{-3} P_{\mathrm{ms}}$ s changes with time according to

$P_{\mathrm{ms}}^{2}(t)=P_{0, \mathrm{~ms}}^{2}+2 \times 10^{-9} t B_{12}^{2}$,

where $P_{0, \mathrm{~ms}}$ is the initial period of the pulsar and $B=10^{12} B_{12} \mathrm{G}$ is the strength of its surface magnetic field. The expending nebula increases the mass from the surrounding medium according to

$M_{\mathrm{Neb}}(t)=M_{0, \mathrm{Neb}}+\frac{4}{3} \pi \rho_{\mathrm{sur}} R_{\mathrm{Neb}}^{3}(t)$,

where $\rho_{\text {sur }}$ is the density of surrounding medium, and $R_{\mathrm{Neb}}(t)$ is the radius of expending envelope at the time, $t$, which depends on the expansion history of the nebula,

$R_{\mathrm{Neb}}(t)=\int_{0}^{t} V_{\mathrm{Neb}}\left(t^{\prime}\right) \mathrm{d} t^{\prime}$

$V_{\mathrm{Neb}}(t)$, and the average density of matter inside the nebula, $\rho_{\mathrm{Neb}}(t)=3 M_{\mathrm{Neb}}(t) / 4 \pi R_{\mathrm{Neb}}^{3}(t)$, have been found numerically by solving the above set of Eqs. (1-5). These parameters are shown in Fig. 1 for different initial periods of the pulsar.

The pulsar loses energy in the form of relativistic wind which extends up to the distance, $R_{\mathrm{sh}}$, at which the pressure of the wind is balanced by the pressure of expending nebula. We obtain the location of $R_{\mathrm{sh}}$ as a function of time by comparing the wind energy flux with the pressure of the outer nebula, which is in turn determined by the supply of magnetic energy to the nebula by the pulsar over its all lifetime (Rees \& Gunn 1974),

$\frac{L_{\mathrm{pul}}(t)}{4 \pi R_{\mathrm{sh}}^{2} c} \approx \frac{\int_{0}^{t} \eta L_{\mathrm{pul}}\left(t^{\prime}\right) \mathrm{d} t^{\prime}}{\frac{4}{3} \pi R_{\mathrm{Neb}}^{3}}$ 

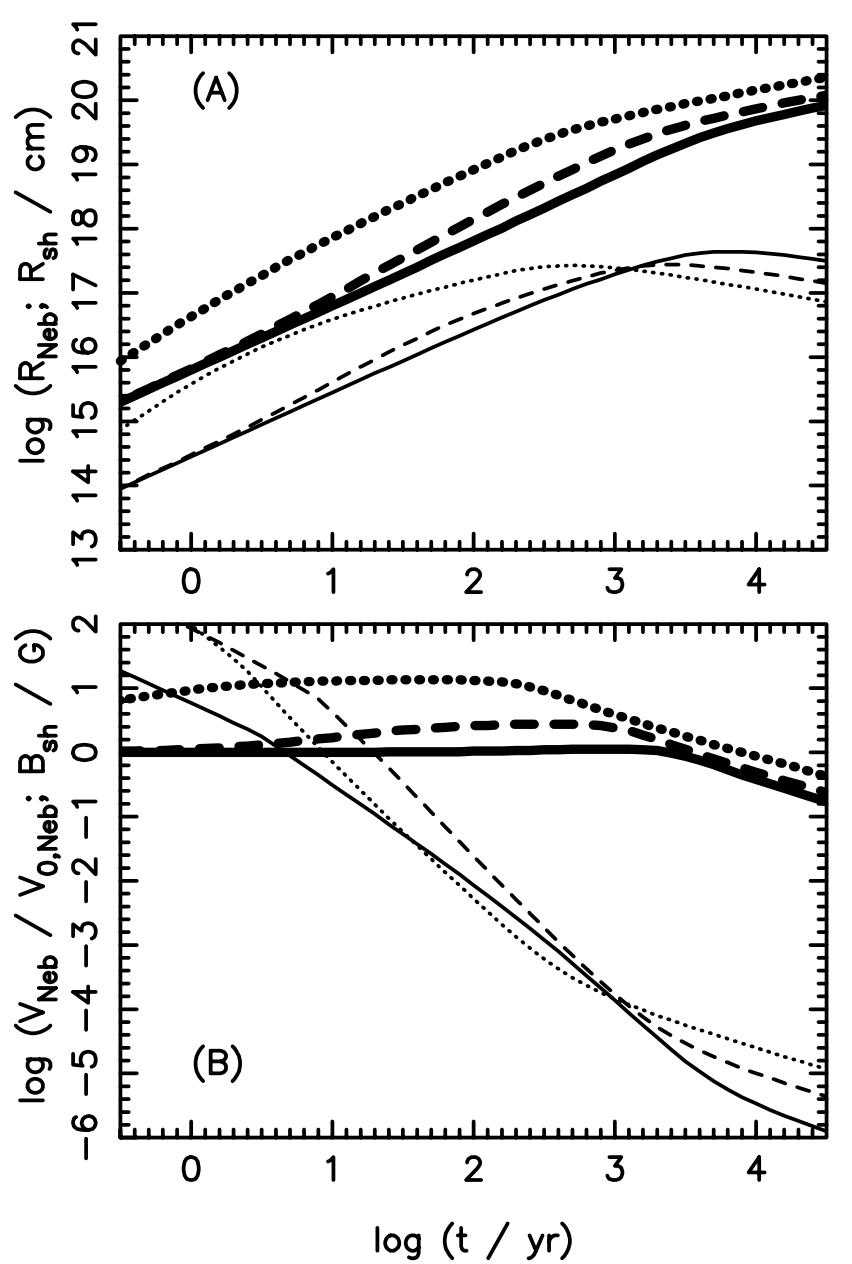

Fig. 1. A) The characteristic distances in the nebula are shown as a function of time: the radius of the nebula $R_{\mathrm{Neb}}$ (thick curves), the radius of the pulsar wind shock $R_{\mathrm{sh}}$ (thin curves), for the pulsar surface magnetic field $5 \times 10^{12} \mathrm{G}$ and its three initial periods: $1 \mathrm{~ms}$ (dotted curves), $5 \mathrm{~ms}$ (dashed curves) and $20 \mathrm{~ms}$ (full curves). The initial expansion velocity of the nebula is $2000 \mathrm{~km} \mathrm{~s}^{-1}$, its mass $4 M_{\odot}$, and the density of surrounding medium is $0.1 \mathrm{~cm}^{-3}$. B) The expansion velocity of the nebula (thick curves) and the strength of the magnetic field at the shock region (thin curves) are shown as a function of time for the same initial parameters of the pulsar and nebula as in $\mathbf{A}$ ).

where $\eta$ is the ratio of the magnetic energy flux to the total energy flux lost by the pulsar, measured at the location of the pulsar wind shock at $R_{\mathrm{sh}}$. The value of $\eta$ changes with the age of the pulsar. It is related to so called "magnetization parameter", $\sigma$, which is the ratio of the magnetic energy flux to the particle energy flux lost by the pulsar by $\eta=\sigma /(1+\sigma)$. The evolution of $\sigma$ with the parameters of the pulsar is found by interpolating between the values estimated for the Crab pulsar, $\sim 0.003$, and for the Vela pulsar, $\sim 1$ (see for details Eq. (16) and below in Bednarek \& Protheroe 2002).

Knowing how the magnetic field depends on the distance from the pulsar, in the pulsar inner magnetosphere and in the pulsar wind zone, we can estimate the strength of the magnetic field after the wind shock from,

$B_{\mathrm{sh}} \approx 3 \sigma B_{\mathrm{pul}}\left(\frac{R_{\mathrm{pul}}}{R_{\mathrm{lc}}}\right)^{3} \frac{R_{\mathrm{lc}}}{R_{\mathrm{sh}}}$,

(7) $\left(\frac{\mathrm{d} E}{\mathrm{~d} t}\right)_{\mathrm{acc}}=\frac{c E}{2 \pi r_{\mathrm{g}} \rho}$, where $R_{\mathrm{pul}}$ and $B_{\mathrm{pul}}$ are the radius and the surface magnetic field of the pulsar.

In our further considerations it is assumed that the typical parameters of the nebula at the moment of supernova explossion are: the expansion velocity of of the bulk matter of the supernova $V_{0, \mathrm{Neb}}=2 \times 10^{3} \mathrm{~km} \mathrm{~s}^{-1}$, and its initial mass $M_{0, \mathrm{SN}}=4 M_{\odot}$ (with good agreement with the parameters derived for the Crab Nebula, see Davidson \& Fesen 1985).

\section{Acceleration of particles by the pulsar}

In this section we define the spectrum of particles which are injected into the nebula. It is assumed that significant part of rotational energy of the pulsar is curried out by iron nuclei, which are extracted from the surface of the neutron star. In fact, the cohesive and binding energy of the iron nuclei on the neutron star surface is not well known. If it is of the order of 2-3 keV (for the magnetic field of a few $10^{12} \mathrm{G}$, see Abrahams \& Shapiro 1991), then the iron nuclei can be thermally emitted for the surface temperature $T \cong 3.5 \times 10^{5} B_{12}^{0.73} \mathrm{~K}$ (Usov \& Melrose 1995). According to the standard cooling model of the neutron stars (Nomoto \& Tsuruta 1987), their surface temperatures are $>10^{6} \mathrm{~K}$ for the age $<10^{5} \mathrm{yrs}$. Therefore, it is possible that the neutron stars in the PWNe inject the iron nuclei. These nuclei can be accelerated in the inner magnetosphere and/or the pulsar wind zone. From normalization to the observations of the Crab pulsar, Arons and collaborators (see Arons 1998) postulate that the Lorenz factors of iron nuclei, which reach the region of the pulsar wind shock, should be

$\gamma_{\mathrm{Fe}} \approx 0.3 Z e \Phi_{\mathrm{open}} / m_{\mathrm{i}} c^{2}$

where $m_{\mathrm{i}}$ and $Z e$ are the mass and charge of the iron nuclei, $c$ is the velocity of light, and $\Phi_{\text {open }}=\sqrt{L_{\mathrm{em}} / c}$ is the total electric potential drop across the open magnetosphere. These nuclei take significant part, $\chi$, of the total energy lost by the pulsar. In the model of Gallant \& Arons (1994), the iron nuclei generate the Alfven waves in the down-stream region of the wind shock, which energy is resonantly transfered to positrons present in the wind, as shown by particle-in-cell simulations by Hoshino et al. (1992). As a result, the positrons obtain close to the power law spectrum with the spectral index $\delta_{1}$ between $E_{1}=\gamma_{\mathrm{Fe}} m_{\mathrm{e}} c^{2}$ and $E_{2} \approx \gamma_{\mathrm{Fe}} A m_{\mathrm{i}} c^{2} / Z$ (see Gallant \& Arons 1994), where $m_{\mathrm{e}}$ and $m_{\mathrm{i}}$ are the electron and ion masses, respectively. The spectrum is normalized to the conversion efficiency of energy from the iron nuclei to the positrons, $\xi$.

For very fast pulsars the magnetic field in the shock region can be high enough to limit the energies of positrons by synchrotron losses. These maximum energies of positrons can be estimated from comparison of the acceleration rate with the synchrotron energy loss rate, $\mathrm{d} E / \mathrm{d} t=\beta U_{\mathrm{B}} E^{2}$, where constant $\beta=4 c \sigma_{\mathrm{T}} /\left(3 m_{\mathrm{e}}^{2}\right), m_{\mathrm{e}}$ is the electron mass, $U_{\mathrm{B}}=B^{2} / 8 \pi$, and $B$ is the magnetic field strength in the acceleration region. The acceleration process discussed by Gallant \& Arons (1994) occurs on a time scale corresponding to a few Larmor radii of particles with the maximum allowed energies, i.e. at a rate 
where $\rho$ is the number of particles Larmor's radii, $r_{\mathrm{g}}=E / e B$. The maximum energy of $\gamma$-ray photons, observed from the Crab Nebula, allows to estimate the value of the parameter $\rho \approx 1$. Therefore, the maximum energies of positrons allowed by the synchrotron losses are

$E_{\mathrm{syn}}^{\max }=\left(\frac{8 \pi e c}{\rho \beta B_{\mathrm{sh}}}\right)^{1 / 2} \approx 24\left(\rho B_{\mathrm{sh}}\right)^{-1 / 2} \mathrm{TeV}$.

We assume that if $E_{2}<E_{\mathrm{syn}}^{\max }$, then the cut-off in the positron spectrum is at $E_{\mathrm{syn}}^{\max }$. The rest of the energy transfered from nuclei to positrons with energies $E_{\mathrm{syn}}^{\max }$. We also postulate the injection of the $e^{ \pm}$pairs into the nebula with energies $E_{1}$, which are accelerated in the pulsar wind. The energy contained in these $e^{ \pm}$pairs is normalized to the part of the energy loss rate of the pulsar equal to $(1-\chi) L_{\text {pul }}$.

The free parameters of considered here model, $\chi$ and $\xi$, can be fixed for the specific PWNa by comparing the calculated synchrotron spectrum with the observations of the PWNa in the radio to $\mathrm{X}$-ray energy range.

\section{The equilibrium spectra of relativistic particles inside the nebula}

In the previous section we defined the spectra of iron nuclei and leptons injected by the pulsar to the nebula as a function of time, measured from the pulsar formation. In order to determine the equilibrium spectra of these particles inside the nebula at a specific time we have to apply numerical approach. This is due to the fact that the processes, which determine the escape and energy losses of injected particles, depend in a complicated way on the parameters of the nebula during its evolution. Below we calculate the equilibrium spectra of different types of nuclei (from disintegration of iron nuclei) and the equilibrium spectra of leptons inside the nebula as a function of time.

\subsection{Spectra of nuclei}

The nuclei injected into the nebula can lose energy as a result of the acceleration of positrons, collisions with the matter of the nebula, and due to adiabatic expansion of the nebula. At the early stage, when the nebula is relatively dense, nuclei also fragment to lighter products due to the collisions with the matter. The most energetic nuclei can also diffuse out of the nebula. All these processes, adiabatic and collisional losses, fragmentation, escape, have been recently studied in detail by Bednarek \& Protheroe (2002) with the purpose to calculate the contribution of pulsar accelerated nuclei to the cosmic rays in the Galaxy. In order to calculate the equilibrium spectra of different types of nuclei inside the nebula at a specific time, we follow the method adopted in Bednarek \& Protheroe (2002), and described in detail in Sects. 4.2 and 4.3 of that paper. The only difference concerns the model for the expansion of the nebula which in the present paper include the effects of energy supplied by the pulsar to the nebula (Sect. 2).

However, in contrary to Bednarek \& Protheroe (2002), who assumed that nuclei are accelerated only by the electric field of the outer gap in the pulsar magnetosphere (Cheng et al. 1986),
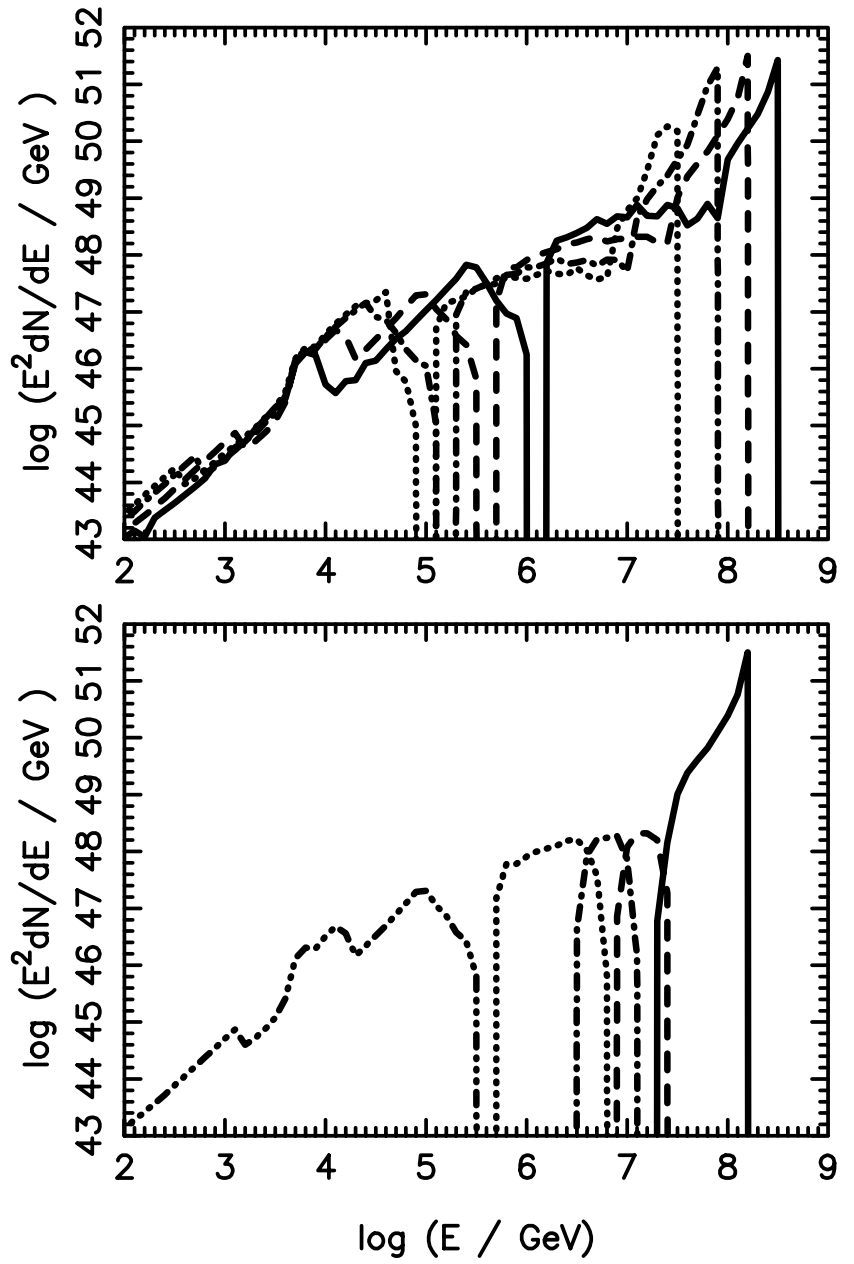

Fig. 2. The equilibrium spectra of nuclei inside the nebula at specific time after the pulsar formation are shown in the upper figure for: $3 \times 10^{2}$ yrs (full curve), $10^{3}$ yrs (dashed), $3 \times 10^{3}$ yrs (dot-dashed), and $10^{4} \mathrm{yrs}$ (dotted). The initial parameters of the pulsar are $P_{\mathrm{o}}=15 \mathrm{~ms}$ and $B=4 \times 10^{12} \mathrm{G}$, the mass of the nebula $4 M_{\odot}$, and its expansion velocity $2000 \mathrm{~km} \mathrm{~s}^{-1}$. The spectra of nuclei with different mass numbers, $\mathrm{A}$, at $10^{3} \mathrm{yrs}$ after the pulsar formation are shown in the bottom figure: A = 1 (dot-dot-dot-dashed curve), 2-10 (dotted), 11-20 (dotdashed), 21-40 (dashed), and 41-55 (full).

we make use of the model proposed by Gallant \& Arons (1994) (see details in Sect. 3 in that paper), in which the nuclei can reach energies corresponding to the significant part of the electric field drop through the pulsar polar cap. Therefore, the iron nuclei, extracted from the neutron star surface, are: (1) at first accelerated in the outer gap where they can lose only a small number of nucleons in collisions with the radiation field of the outer gap (see Sect. 4.1 in Bednarek \& Protheroe 2002 and Bednarek \& Protheroe 1997) and, (2) farther accelerated in the pulsar wind zone to the Lorentz factors $\gamma_{\mathrm{Fe}}(\mathrm{Eq} .(8))$, determined by the pulsar parameters.

As an example, in Fig. 2 we show the equilibrium spectra of nuclei inside the nebula for the case of the pulsar with initial parameters $P_{\mathrm{o}}=15 \mathrm{~ms}$ and $B=4 \times 10^{12} \mathrm{G}$, the mass of the nebula $4 M_{\odot}$, and its expansion velocity $2000 \mathrm{~km} \mathrm{~s}^{-1}$ (as expected for the Crab pulsar). The upper figure shows the spectra of nuclei at different time after the explosion of supernova. 
As expected the maximum energies and the numbers of relativistic nuclei inside the nebula decrease with time due to the adiabatic energy losses and escape of the most energetic nuclei from the nebula. However the spectra of nuclei at lower energies (lighter nuclei) do not change significantly. The bottom figure shows the spectra of nuclei with different mass numbers at $10^{3}$ years after the supernova explosion. The spectrum of nuclei is dominated by the heavy elements from the iron group. These nuclei have been mainly injected into the nebula when it becomes transparent for disintegration of nuclei. This typically happens at several to a few tens of years after the supernova explosion.

\subsection{Spectra of leptons}

Positrons injected into the medium of expending supernova remnant suffer energy losses mainly on radiation processes, bremsstrahlung, synchrotron, and the inverse Compton, and due to the expansion of the nebula. The rate of their energy losses can be described by

$-\frac{\mathrm{d} E}{\mathrm{~d} t}=\left(\alpha_{1}+\alpha_{2}\right) E+\left(\beta_{1}+\beta_{2}\right) E^{2} \quad \mathrm{GeV} \mathrm{s}^{-1}$,

where $\alpha_{1} \approx m_{\mathrm{p}} N /\left(m_{\mathrm{e}} X\right) \approx 7.8 \times 10^{-16} \mathrm{~N} \mathrm{~s}^{-1}$ describes the bremsstrahlung losses, where $m_{\mathrm{p}}$ is the proton mass, $X$ is the radiation length and $N$ is the density of the medium in $\mathrm{cm}^{-3} ; \alpha_{2}=$ $V_{\mathrm{Neb}}(t) / R_{\mathrm{Neb}}(t)$ describes the adiabatic losses due to the expansion of the nebula (Longair 1981); $\beta_{1} \approx 4 c \sigma_{\mathrm{T}} U_{\mathrm{B}} /\left(m_{\mathrm{e}}^{2} c^{5}\right) \approx$ $2.55 \times 10^{-6} B^{2} \mathrm{GeV}^{-1} \mathrm{~s}^{-1}$ the synchrotron energy losses, where $\sigma_{\mathrm{T}}$ is the Thomson cross section, $U_{\mathrm{B}}$ is the magnetic field energy density and $B$ is the magnetic field in $\mathrm{G}$; and $\beta_{2} \approx$ $4 c \sigma_{\mathrm{T}} U_{\text {rad }} /\left(m_{\mathrm{e}}^{2}\right) \approx 1.05 \times 10^{-7} U_{\text {rad }} \mathrm{GeV}^{-1} \mathrm{~s}^{-1}$ the ICS losses in the Thomson regime. $U_{\text {rad }}$ is the energy density of different types of soft radiation inside the nebula, i.e. the synchrotron radiation created by leptons in the magnetic field of the nebula, the MBR, and the infrared photons produced by the dust inside the nebula. The energy losses of leptons on the ICS in the Klein-Nishina regime can be safely neglected in respect to the synchrotron energy losses. The density of synchrotron radiation depends on the spectrum of positrons which is in turn determined by their energy losses at the earlier phase of expansion of the nebula.

The coefficients, $\alpha_{1}, \alpha_{2}, \beta_{1}$, and $\beta_{2}$, depend on time in a complicated way due to the changing conditions in the expending nebula (magnetic field, density of matter and radiation). Therefore, Eq. (11) cannot be integrated analytically. In order to determine the energies of leptons, $E$, inside the nebula at a specific time, $t_{\mathrm{obs}}$, which have been injected with energies, $E_{\mathrm{o}}$, at an earlier time, $t^{\prime}$, we use the numerical approach. The equilibrium spectrum of leptons is then obtained by integrating their injection spectra over the age of the nebula

$\frac{\mathrm{d} N\left(t_{\mathrm{obs}}\right)}{\mathrm{d} E}=\int_{0}^{t_{\mathrm{obs}}} J\left(t^{\prime}\right) \frac{\mathrm{d} N}{\mathrm{~d} E_{\mathrm{o}} \mathrm{d} t} \mathrm{~d} t$,

where $\mathrm{d} N / \mathrm{d} E_{\mathrm{o}} \mathrm{d} t$ is the injection spectrum of positrons at the time $t$, defined in Sect. 3, $t^{\prime}=t_{\mathrm{obs}}-t$, and the jacobian $J\left(t^{\prime}\right)=E_{\mathrm{o}} / E$.

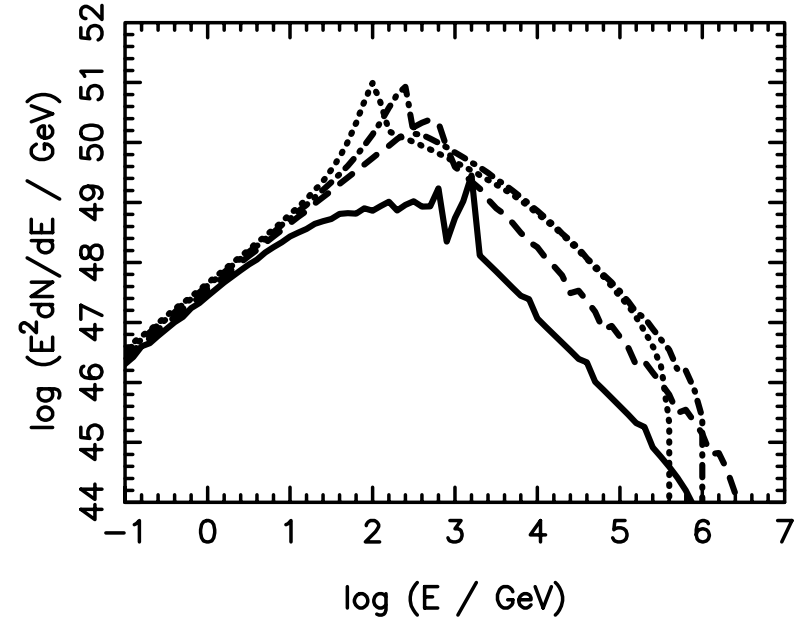

Fig. 3. The equilibrium spectra of positrons at the time $3 \times 10^{2}$ yrs (full curve), $10^{3}$ yrs (dashed), $3 \times 10^{3}$ yrs (dot-dashed), and $10^{4}$ yrs (dotted) after the pulsar formation. Leptons are injected in two acceleration processes: 1) the positrons with the power law spectrum and spectral index 2.5, and 2) monoenergetic $e^{ \pm}$, accelerated together with the iron nuclei in the pulsar wind zone to the Lorentz factors $\gamma_{\mathrm{Fe}}$. It is assumed that nuclei take $\chi=0.85$ of the rotational energy lost by the pulsar and accelerates positrons with the efficiency $\xi=0.5$. The rest of available energy, $1-\chi=0.15$, is transfered to the monoenergetic $e^{ \pm}$pairs.

The example spectra of leptons inside the nebula at the specific time after explosion of supernova are shown in Fig. 3, assuming that leptons are injected into the nebula with the two component spectrum: 1) the power law spectrum normalized to the pulsar energy loss rate, $\chi=0.85$, and efficiency of energy conversion from nuclei into positrons, $\xi=0.5$, and the spectral index of the positrons equal to $\alpha=2.5 ; 2$ ) The monoenergetic $e^{ \pm}$pairs accelerated in the pulsar wind zone to energies $E_{1}$. The density of leptons inside the nebula is lower when the nebula is younger in spite of the higher rate of injected leptons. This is due to the much higher efficiency of energy loss processes of positrons in young nebula when the magnetic field and the radiation energy densities are much larger. That's why, the equilibrium spectra of positrons cuts-off at lower energies when the nebula is young. However, at later stage of the nebula, the cut-off in the spectrum of leptons occurs again at the lower energies due to the lower energies of injected leptons. The maximum in the equilibrium spectrum of leptons shifts to higher energies at the early stage, and then drops to lower energies for the nebulae with the age larger than $\sim 10^{3}$ years.

\section{Production of radiation inside the Nebula}

The knowledge on the equilibrium spectra of relativistic particles (leptons and nuclei) as a function of time after supernova explosion allows us to calculate the photon spectra produced inside the nebula by these particles in different radiation processes. Leptons produce photons mainly on synchrotron, bremsstrahlung, and ICS processes. Nuclei produce $\gamma$-rays in collisions with the matter via decay of neutral pions. We neglect the radiation produced by the secondary $e^{ \pm}$pairs from decay of charged pions, since their contribution to the equilibrium spectrum of leptons inside the nebula is negligible in respect to 

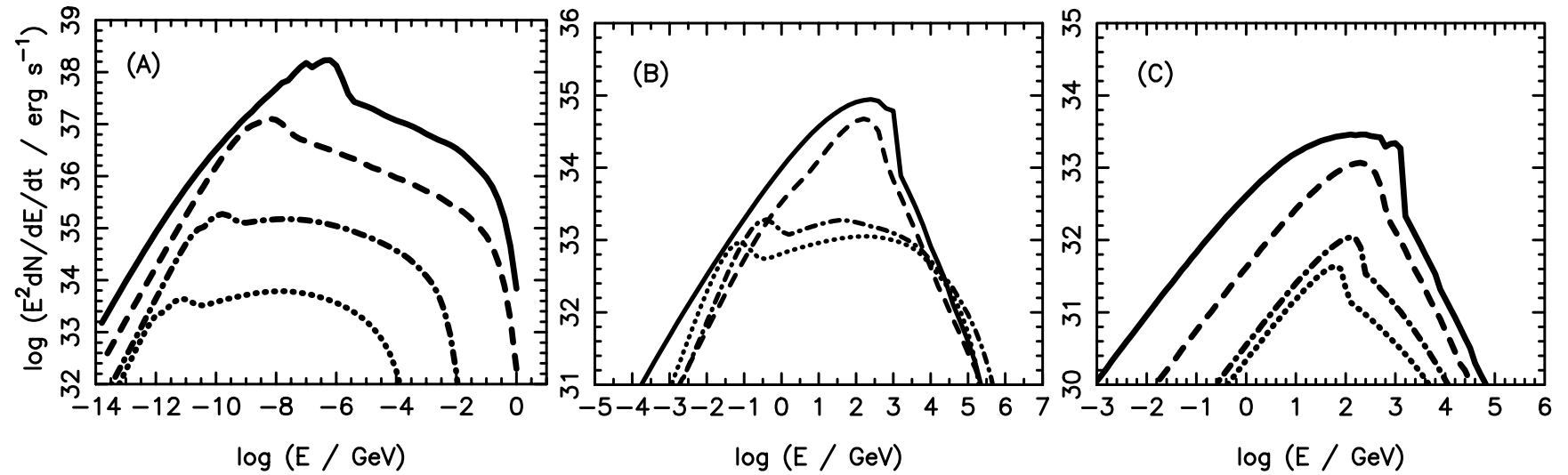

Fig. 4. Photon spectra produced by leptons in synchrotron A), inverse Compton B), and bremsstrahlung $\mathbf{C}$ ) processes, at the time $3 \times 10^{2}$ yrs (full curves), $10^{3} \mathrm{yrs}$ (dashed), $3 \times 10^{3} \mathrm{yrs}$ (dot-dashed), and $10^{4} \mathrm{yrs}$ (dotted) after the supernova explosion. Positrons are injected into the nebula with the power law spectrum and spectral index 2.5 between $E_{1}$ and $E_{2}$ (see text), normalized to the pulsar energy loss rate with $\chi=0.85$, and the acceleration efficiency $\xi=0.5$. The monoenergetic $e^{ \pm}$pairs are injected with energies $E_{1}$.

the contribution from primary positrons, accelerated directly in resonant interactions with heavy nuclei and in the pulsar wind zone.

\subsection{Radiation from leptons}

The conditions in the expanding nebula, i.e. the magnetic and radiation fields and the density of matter, change significantly with time in a different manner. Thus, the relative importance of specific radiation processes has to change as well. At the early stage of expansion of the nebula, the synchrotron energy losses of leptons dominate over the ICS and the bremsstrahlung energy losses. Therefore, most of the energy of leptons is radiated in the low energy range. When the nebula becomes older, the energy density of the synchrotron radiation inside the nebula decreases but the energy density of microwave background radiation (MBR) remains constant. Therefore, relative importance of the ICS losses increases in respect to the synchrotron energy losses. The energy losses of leptons on synchrotron process and bremsstrahlung process can be simply compared for known parameters of the nebula at a specific time. The bremsstrahlung process dominates over the synchrotron process for leptons with energies,

$$
E<\alpha_{1} / \beta_{1} \approx 4.1 \times 10^{-10} N B^{-2} \mathrm{GeV} .
$$

For example, if the age of the nebula is $10^{3} \mathrm{yrs}$ (the Crab Nebula), then the density of matter is $N \sim 10 \mathrm{~cm}^{-3}$ and the magnetic field $B \sim 10^{-4} \mathrm{G}$. For these parameters, the synchrotron energy losses of leptons dominate over their bremsstrahlung energy losses for energies as low as $\sim 0.4 \mathrm{GeV}$. Therefore, if there exists large population of hundred MeV leptons inside the Crab Nebula, they should radiate bremsstrahlung photons with the $\mathrm{MeV}$ energies.

In order to determine precise contribution of these three processes, we calculate the photon spectra produced by leptons with the equilibrium spectrum, obtained as shown in the previous section. As an example, in Fig. 4 we show the photon spectra from these three process at a specific time after supernova explosion for the equilibrium spectra of leptons obtained for the initial parameters of the pulsar and nebula as defined in Fig. 3. It is assumed that leptons produce the IC $\gamma$-rays by scattering the synchrotron and MBR photons. Note that the synchrotron spectra from the nebula strongly decrease with time, and the contribution of the bremsstrahlung spectra to the $\gamma$-ray energy range is always at least an order of magnitude lower than the contribution of the IC spectra.

\subsection{Gamma-rays from hadrons}

The nuclei with the equilibrium spectra interact with the matter of the nebula and produce $\gamma$-rays via decay of pions. We calculate $\gamma$-ray spectra from hadronic interactions applying the scaling break model proposed by Wdowczyk \& Wolfendale (1987). As an example, we show in Fig. 5 the spectra of $\gamma$-rays produced at different time after the supernova explosion. The initial parameters of the pulsar and the supernova are taken as in the example shown for the photon spectra produced by leptons. The intensities of the $\gamma$-ray spectra decrease with the age of the nebula due to the lower densities of matter and relativistic nuclei inside the nebula. This decrease is much faster than observed in the case of the $\gamma$-ray spectra produced by leptons in ICS process. Therefore, the contribution of $\gamma$-rays from hadronic processes is relatively less important for older nebulae. The $\gamma$-ray spectra from hadronic collisions shift to lower energies due to the adiabatic energy losses of nuclei, more efficient escape of higher energy nuclei from the nebula, and lower energies of freshly injected nuclei inside the nebula by older pulsars.

\section{Application to specific supernova remnants}

The radiation model, considered above, is at first compared with the observations of the best investigated WPNa, i.e. the Crab Nebula. Our strategy is to fix some free parameters of the model based on the comparison of the observed radio up to the TeV $\gamma$-ray spectrum with our calculations of the synchrotron, ICS, and $\gamma$-ray spectrum from decay of pions. Trying to keep constant as many as possible parameters derived for the 


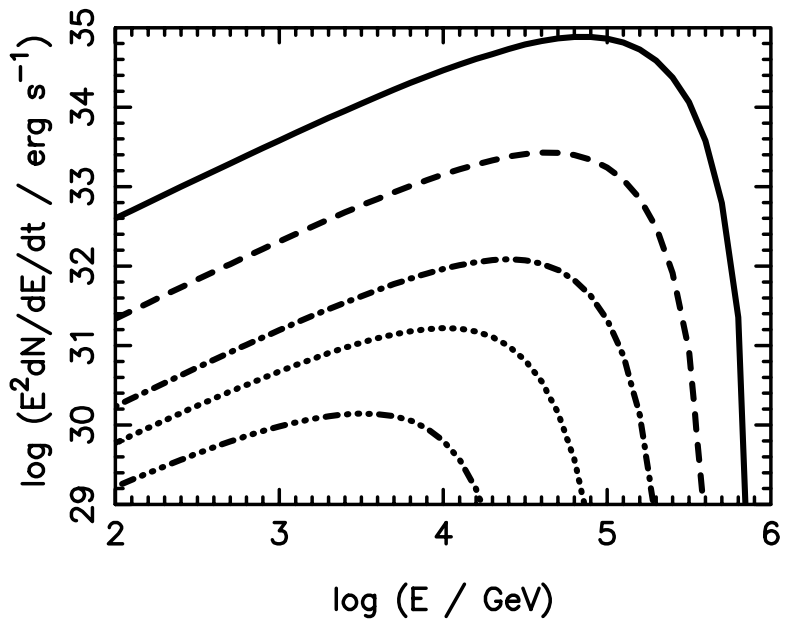

Fig. 5. Spectra of gamma-rays produced in the interactions of nuclei, with the equilibrium spectra shown in Fig. 2, with the matter of the nebula at a specific time after the pulsar formation: $3 \times 10^{2} \mathrm{yrs}$ (full curve), $10^{3}$ yrs (dashed), $3 \times 10^{3}$ yrs (dot-dashed), $10^{4}$ yrs (dotted), and $3 \times 10^{4} \mathrm{yrs}$ (dot-dot-dot-dashed). The initial parameters of the pulsar and the nebula are as in Figs. 2 and 3.

Crab Nebula, we calculate the high energy photon spectra for the cases of other PWNe, which were also reported at the TeV $\gamma$-rays, i.e. Vela pulsar and the nebula around PSR 1706-44. Interestingly, the observed parameters of these pulsars and nebulae can be obtained assuming these same initial parameters as found for the Crab pulsar, i.e. it seems that these two pulsars and nebulae are on these same evolutionary path as the Crab pulsar and nebula. Using the experience obtained from modelling the nebulae with known $\gamma$-ray emission, we use this model to predict the level of $\mathrm{TeV} \gamma$-ray emission from the nebulae discovered around other energetic pulsars: J0205+6449 in the nebula 3C 58, and PSR 1951+32 in the nebula CTB80.

\subsection{The Crab Nebula}

The Crab Nebula is the only WPNa that has been detected in the all energies of the electromagnetic spectrum, starting from the radio up to a few tens of $\mathrm{TeV} \gamma$-rays. The Crab Nebula photon spectrum consists of two broad bumps: first one, from radio up to $\mathrm{MeV} \gamma$-rays, with the spectrum described by simple power law with the index $\sim 2.25$ from optical to $\mathrm{MeV} \gamma$-rays; and the second one, from a hundred $\mathrm{MeV}$ up to $\sim 50 \mathrm{TeV}$, described also by the power law spectrum with the spectral index $\sim 2.5$ between 0.1-50 TeV (see Fig. 6).

As in other works, we interpret the first broad bump in the Crab Nebula spectrum as synchrotron emission produced by leptons inside the nebula. The second bump is interpreted as the ICS of soft photons inside the nebula by these same population of leptons and by self-consistently calculated $\gamma$-ray emission from decay of charged pions, produced in collisions of nuclei with the matter of the nebula. In order to perform calculations we have to fix at first the initial parameters of the Crab pulsar and nebula. The observed period, $33.4 \mathrm{~ms}$, and the age of the nebula, $950 \mathrm{yr}$, are consistent with the Crab pulsar initial period of $15 \mathrm{~ms}$ and its surface magnetic field of $3.8 \times 10^{12} \mathrm{G}$. The
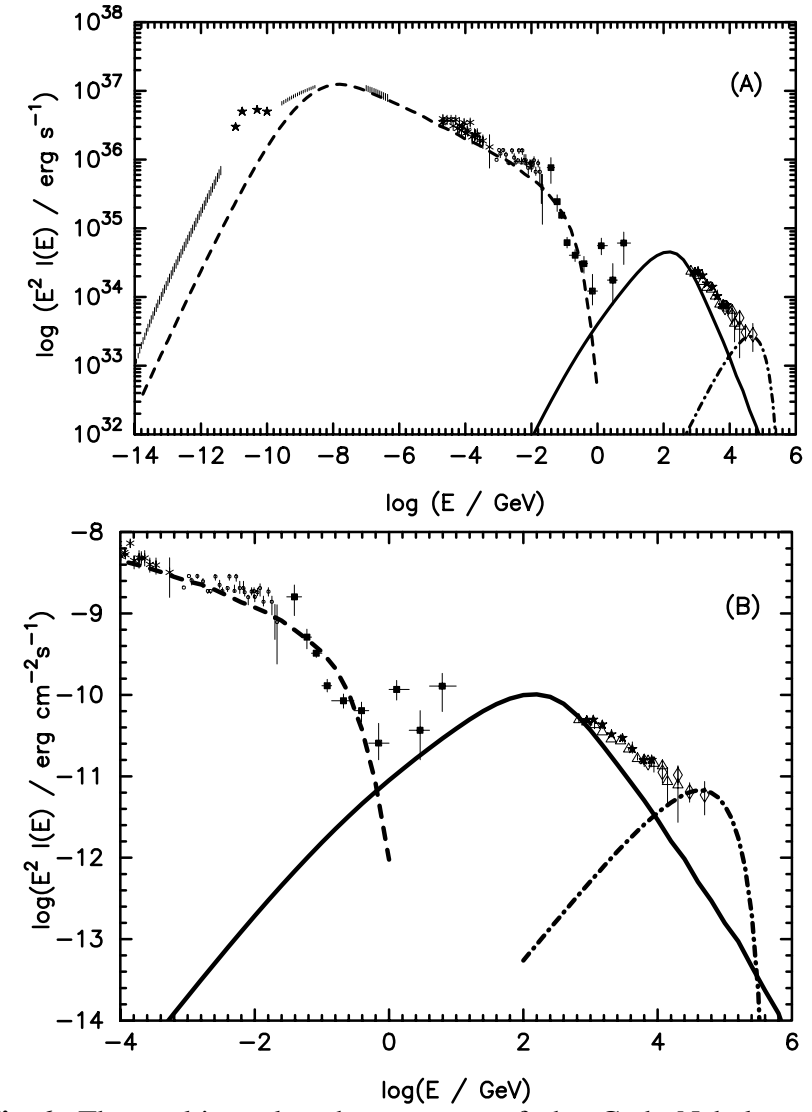

Fig. 6. The multiwavelength spectrum of the Crab Nebula compared with the spectrum calculated in terms of the hadronic-leptonic model A). The details of the Crab Nebula spectrum in the $\gamma$-ray energy range B). Leptons are injected into the nebula with the power law spectrum (positrons accelerated in the interactions with iron nuclei) and spectral index $\alpha=2.5$ betwen $E_{1}$ and $E_{2}$. It is assumed that the iron nuclei take $\chi=0.85$ of the total rotational energy lost by the pulsar. A part of the energy of the iron nuclei, $\xi=0.5$, is converted into relativistic positrons. The rest of the pulsar rotational energy, $1-\chi$, is carried out by the monoenergetic $e^{ \pm}$pairs with energies $E_{1}$. The synchrotron and inverse Compton spectra (comptonization of synchrotron, MBR, and infrared photons), produced by leptons inside the nebula, are shown by the dashed and full curves, respectively. The $\gamma$-ray spectrum from decay of pions, produced by hadrons inside the nebula, is shown by the dot-dashed curve. The other parameters of the pulsar and nebula are given in the text.

observed parameters of the Crab Nebula, the radius of 2-3 pc for the distance $1830 \mathrm{pc}$, the expansion velocity $\sim 2000 \mathrm{~km} \mathrm{~s}^{-1}$, and the mass of the Crab Nebula equal to $\sim 4 M_{\odot}$ (Davidson \& Fesen 1985), are consistent with the present age of the pulsar. We assume that the initial expansion velocity of the bulk matter of the nebula is very close to the observed velocity since the nebula is still in the free expansion phase for the typical density of the surrounding medium $0.3 \mathrm{~cm}^{-3}$.

For the above initial parameters of the Crab pulsar and nebula we have performed a sequence of calculations of the expected broad range spectra in order to obtain good fit to the observed spectrum. As described in Sect. 3, the injection spectrum of leptons consists of two power laws. Above the energy $E_{1}$, the spectral index has to be $\delta_{1}=2.5$, in order to be consistent with the observed synchrotron spectrum with the 
spectral index in the broad energy range equal to $\sim 2.25$. If the iron nuclei take, $\chi=0.85$, of the total energy lost by the Crab pulsar, then the calculated synchrotron emission is consistent with the observed emission (see dashed curve in Fig. 6), for the efficiency of energy conversion from the iron nuclei to the positrons equal to $\xi=0.5$. We assume, moreover, that the rest of energy lost by the pulsar, $1-\chi$, is curried out by the monoenergetic $e^{ \pm}$pairs with the Lorentz factors of the iron nuclei and energies $E_{1}$. For these parameters we can fit the observed synchrotron spectrum in the broad energy range. However, the calculations do not give correct description of the lowest energy part of the spectrum (below optical). This is due to the assumption that all $e^{ \pm}$pairs are accelerated in the pulsar wind to these same monoenergetic energies equal to $E_{1}$. In a more realistic model, the pairs should be accelerated in the pulsar wind to different maximum energies, depending on their injection from different parts of the light cylinder. As a consequence, the $e^{ \pm}$pairs should be injected into the nebula with some spectrum which shape is not precisely known by the theory.

We also calculate the ICS spectrum from the Crab Nebula, applying that the soft photon field for leptons is created by the synchrotron photons produced by this same leptons, microwave background radiation (MBR), and the thermal infrared photons, emitted by the dust inside the nebula with the energy density equal to the energy density of the MBR and the temperature $100 \mathrm{~K}$ (see Aharonian \& Atoyan 1995). The reasonable consistency with the observed level of $\gamma$-ray emission from the Crab nebula at $\sim 1 \mathrm{TeV}$ is obtained if we assume that the energy density of synchrotron photons is $\sim 20$ times higher in the region where the most of the IC photons are produced. In another words the effective volume of synchrotron emission has to be 20 times lower than the whole volume of the nebula. In fact, leptons are not injected into the nebula symmetrically but at some range of angles close to the equatorial plane of the rotational axis of the pulsar as suggested by the observations of the inner X-ray torus.

The calculated ICS spectrum is too steep and does not fit correctly the $\gamma$-ray spectrum observed from the Crab Nebula above a few TeV (see full curve in Fig. 6). However, calculated selfconsistently the spectrum of $\gamma$-ray photons from decay of pions produced by heavy nuclei, which accumulate inside the nebula, fits very well the $\gamma$-ray emission at $\sim 10 \mathrm{TeV}$ (see dotdashed curve in Fig. 6). Note that $\gamma$-ray emission from nuclei does not depend on any additional free parameters since in the considered here model the numbers of relativistic nuclei and leptons are strictly connected.

\subsection{The Vela Supernova Remnant}

The observations of the Vela pulsar performed with the large field and small field detectors show two different spectral shapes in the X-ray energy range. The large field instruments, OSO-8 (Pravdo et al. 1978) and HEAO A-4 (Levine et al. 1984), observe steep emission from the whole Vela Supernova Remnant (SNR), which dominates at energies below $\sim 10 \mathrm{keV}$. On the other hand, the small field detectors, Einstein (Harnden et al. 1985), EXOSAT (Ögelman \& Zimmermann 1989), ROSAT (Ögelmanan et al. 1993), and
Birmingham Spacelab 2 (Willmore et al. 1992), observe flatter emission with the differential spectrum and average spectral index $\sim 1.7$. This spectrum is consistent with the observations of Vela supernova remnant by OSSE between $44 \mathrm{keV}$ and $370 \mathrm{keV}$ (De Jager et al. 1996). The discrepancy between results reported by the large and small field detectors can be explained by assuming that the X-ray spectrum of the Vela compact nebula extends to higher energies and is flatter than the emission observed from the Vela supernova remnant. The unpulsed $\mathrm{TeV} \gamma$-ray emission has been also reported from the direction of the Vela pulsar at energies $>2.5 \mathrm{TeV}$ (Yoshikoshi et al. 1997). At lower energies, only the upper limits are available in the EGRET energy range (Kanbach et al. 1994) and above $300 \mathrm{GeV}$ (Chadwick et al. 2000).

The Vela pulsar is surrounded by the compact non-thermal nebula with the radius of $\sim 7^{\prime}$ and the extended Vela SNR with the radius of $\sim 3.5^{\circ}$. The observed parameters of the pulsar allow us to estimate its surface magnetic field, $4.5 \times 10^{12} \mathrm{G}$, and the characteristic age of $11300 \mathrm{yrs}$. We model the Vela SNR assuming that the initial parameters of this object were similar to the Crab Nebula, i.e. the initial pulsar period $15 \mathrm{~ms}$, the mass of Vela SNR $4 M_{\odot}$ and the expansion velocity $2000 \mathrm{~km} \mathrm{~s}^{-1}$. The distance to the Vela pulsar is taken $\sim 300$ pc (Caraveo et al. 2001), although the older literature suggested the value of 500 pc (e.g. Cha et al. 1999). For these parameters of the pulsar and nebula, we estimate its real age $\sim 6000 \mathrm{yrs}$, from the consistency with the observed dimension of the nebula and the observed period of the Vela pulsar.

We consider the radiation processes around the Vela pulsar as a two stage process. At first leptons are injected into the inner nebula with a relatively small size, $\sim 0.15 \mathrm{pc}$. They move through the Vela compact nebula with high velocity (about one third of the velocity of light) losing only part of their initial energies. The average magnetic field in the Vela WPN is estimated on $4 \times 10^{-5} \mathrm{G}$. After that, leptons are injected into the large scale Vela SNR, where the magnetic field is close to that in the interstellar medium, $\sim 5 \times 10^{-6} \mathrm{G}$. The model with above magnetic field strengths can explain the different spectra observed by the large and small field X-ray detectors.

In order to fit the expected X-ray and $\gamma$-ray spectra from the Vela WPNa by the synchrotron and IC spectra calculated in terms of such a model, we assume that the iron nuclei take similar part of the energy lost by the pulsar as in the case of the Crab pulsar, i.e. $\chi=0.85$. However, to obtain the correct level of synchrotron emission from the inner and the outer nebulae, we were forced to assume that the average acceleration efficiency of positrons by the nuclei is much lower than in the case of the Crab pulsar and equal to $\xi=0.07$. In Fig. 7, the results of calculations of the synchrotron spectra and the IC spectrum are compared with the observations of the Vela PWNa in the X-rays, with the upper limits from the EGRET observations (Kanbach et al. 1994), and with the TeV $\gamma$-ray energy range (Yoshikoshi et al. 1997). The emission from the extended Vela SNR fits well to the total spectrum from the Vela nebula below $\sim 10 \mathrm{keV}$ and is responsible for the $\gamma$-ray emission observed in the TeV energy range. Note that the IC spectra are produced mainly due to the scattering of the MBR. The synchrotron photons can be neglected for nebulae with the 


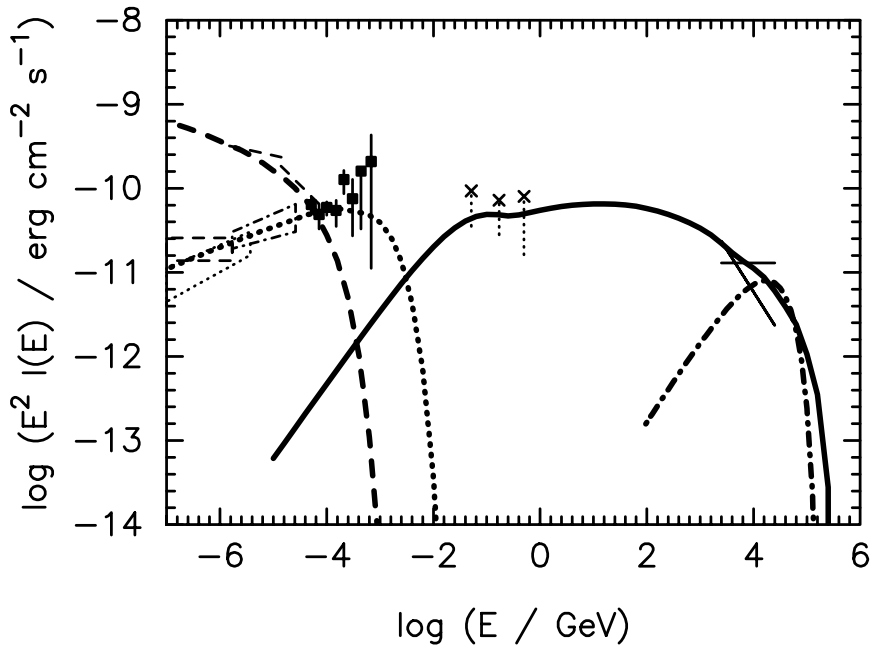

Fig. 7. The high energy spectrum observed from the compact nebula around the Vela pulsar and from the Vela SNR (see text for details) is compared with the spectrum calculated in terms of the discussed hadronic-leptonic model. Relativistic leptons produce synchrotron spectrum during propagation through the compact nebula with the strong magnetic field (dotted curve) and after that, in the extended Vela SNR (dashed curve). The IC spectrum, produced by leptons in the Vela SNR due to the comptonization of the MBR, is shown by the full curve. The $\gamma$-ray spectrum from decay of pions, produced by nuclei inside the Vela SNR, is shown by the dot-dashed curve. The initial parameters of the pulsar and supernova are as in the case of the Crab Nebula (accept the magnetic field of the pulsar) and the real age of the nebula equal to $6000 \mathrm{yrs}$.

age of the Vela pulsar. On the other hand, the emission from the compact nebula dominates in the hard X-rays (between $\sim 10 \mathrm{keV}$ and $\sim 1 \mathrm{MeV}$ ). The contribution of leptons from the compact nebula to the high energy $\gamma$-ray spectrum is negligible since we assumed that they move relatively fast through the inner nebula. We have also calculated the $\gamma$-ray spectrum from decay of pions, produced by heavy nuclei injected by the pulsar (dot-dashed curve in Fig. 7). Their contribution to the total $\gamma$-ray spectrum observed from the Vela SNR above $\sim 10 \mathrm{TeV}$ is still comparable to the IC spectrum, but is relatively lower than in the case of the Crab Nebula.

\subsection{The Nebula around PSR 1706-44}

The pulsar PSR 1706-44 has been associated with the supernova remnant SNR G 343.1-2.3 on the basis of radio observations (McAdam et al. 1993; Dodson \& Golap 2002), although there are also some distance inconsistencies between these two objects (Frail et al. 1994). PSR 1706-44 shows close similarities to the Vela pulsar and its SNR since these pulsars have similar characteristic ages and present periods. PSR 1706-44 is also immersed in a very compact nebula with a radius $\sim 0.1-0.3 \mathrm{pc}$ (Becker et al. 1995). The spectrum of this nebula in the energy range $0.1-2.4 \mathrm{keV}$ has the differential photon index $2.4 \pm 0.6$, and the spectrum reported in the higher energy range $(0.7-10 \mathrm{keV})$ has the index 1.7 (ROSAT and ASCA observations, Finley et al. 1998). The ROSAT upper limit on the flux from the supernova remnant SNR G 343.1-2.3 is $9 \times 10^{-13} \mathrm{erg} \mathrm{cm}^{-2} \mathrm{~s}^{-1}$, assuming the spectrum with the differential photon index equal to 2 (Becker et al. 1995). Thus the spectral features of the emission from the compact and extended nebulae are similar to this observed in the nebulae surrounding the Vela pulsar. However the intensity of this X-ray emission is on much lower level (see Figs. 6 and 7 for comparison), which can be only partially explained by diffrent distances to these sources. The $\mathrm{TeV} \gamma$-ray emission has been also detected from the region of $<3$ pc around PSR 1706-44 (Kifune et al. 1995; Chadwick et al. 1998), with the intensity similar to that observed from the Vela nebula. Therefore, the spectral features of these two objects differ significantly, since if we take into account the difference in distances to these objects, $\sim 300$ pc to the Vela pulsar, and $1.8 \mathrm{kpc}$ to PSR 1706-44 (Taylor $\&$ Cordes 1993), PSR 1706-44 has much weaker X-ray emission and much stronger $\mathrm{TeV} \gamma$-ray emission.

As in the previous modelling, we assume that the initial period of this pulsar is also $15 \mathrm{~ms}$ and the expansion velocity of the nebula $2000 \mathrm{~km} \mathrm{~s}^{-1}$. For the above parameters of the pulsar and nebula, the consistency with the observed period of the pulsar and dimension of the nebula is reached for the real age of this object equal to $16000 \mathrm{yrs}$. In fact, due to the lower surface magnetic field of PSR1706-44, equal to $3.1 \times 10^{12} \mathrm{G}$, and longer present period, $102 \mathrm{~ms}$, the real age of the pulsar has to be closer to its characteristic age $17400 \mathrm{yrs}$, than in the case of the Vela pulsar.

Now we are ready to calculate the expected spectrum from this nebula. Lower level of the synchrotron emission from the supernova remnant of the pulsar PSR 1706-44 suggest that the acceleration efficiency of leptons has to be lower than in the case of the Vela pulsar. The consistency with the X-ray emission from the nebula, reported by ASCA, is obtained for the efficiency of lepton acceleration $\xi=0.02$, with the other model parameters fixed as in the case of the Vela and the Crab Nebulae (see thick dashed curve in Fig. 8). However, the $\gamma$-ray emission from ICS of the diffusive low energy radiation (mainly MBR) by leptons is by a factor of a few below the reported TeV $\gamma$-ray flux (see thick full curve in Fig. 8). The $\gamma$-rays produced in the interactions of nuclei with the matter of the nebula can not explain the level of $\mathrm{TeV} \gamma$-ray emission as well (dot-dashed curve in Fig. 8).

The IC TeV emission could be enhanced if the additional source of soft radiation or high density matter is present in/or close to the nebula. As already discussed in Sect. 6.1, additional thermal emission with characteristic temperature $\sim 100 \mathrm{~K}$ is observed from the Crab Nebula. In the case of nebula around PSR 1706-44, the energy density of this infrared radiation should be about a factor $\sim 5$ times higher than the MBR, in order to produce observable flux in the $\mathrm{TeV} \gamma$-rays. We show the results of such calculations assuming that the temperature of this thermal radiation is $20 \mathrm{~K}$ (see thin dashed and full curves in Fig. 8). The required acceleration efficiency of leptons in this case is $\xi=0.08$. However the presence of such strong infrared field inside the nebula is unclear. Finley et al. (1998) suggest that likely candidates for such seed photons are IR background photons in the Galactic plane. 


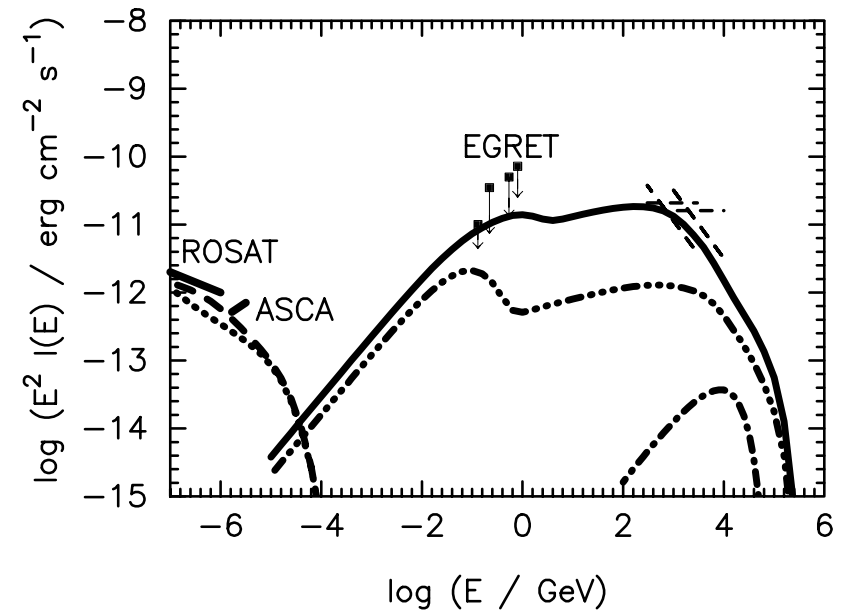

Fig. 8. The high energy spectrum from the nebula (G 343.1-2.3) around the pulsar PSR 1706-44 (see text for details) is compared with the synchrotron spectrum and the IC spectrum, produced by positrons accelerated in interactions with nuclei and $e^{ \pm}$pairs accelerated in the pulsar wind zone. The synchrotron and $\gamma$-ray spectra calculated under the assumption that leptons comptonize only the MBR are shown by the dashed and dot-dot-dot-dashed curves, respectively. These spectra obtained with the additional strong infrared field inside the nebula with the temperature $20 \mathrm{~K}$ are shown by the dotted and full curves, respectively. The initial parameters of the pulsar and the supernova are as in the case of the Crab Nebula. The surface magnetic field of the pulsar is $3.1 \times 10^{12} \mathrm{G}$, and its age, $16000 \mathrm{yrs}$. The $\gamma$-ray spectrum from decay of pions is shown by the dot-dashed curve.

\subsection{The Nebula MSH15-52 around PSR 1509-58}

The supernova remnant MSH15-52, associated with the pulsar PSR 1509-58, has complex structure observed by many experiments in the radio and X-ray bands. The multiwavelenght spectrum of this nebula (see Fig. 9), extends from the radio (data from du Plessis et al. 1995) through the X-rays (ROSAT - Trussoni et al. 1996, Beppo SAX - Mineo et al. 2001, RTXE - Marsden et al. 1997) up to the TeV $\gamma$-rays (Sako et al. 2000). The pulsar and its nebula are sometimes identified with the historical supernova SN 185 (Thorsett 1992). The distance to this object is put in the range $4.2 \mathrm{kpc}$ (kinematics of $\mathrm{H} \mathrm{I}$, Caswell et al. 1975) up to $5.9 \mathrm{kpc}$ (dispersion measure, Taylor \& Cordes 1993). We apply the value of $5.2 \mathrm{kpc}$ derived by Gaensler et al. (1999). The present period of the pulsar is $\sim 150 \mathrm{~ms}$, its surface magnetic field is $1.5 \times 10^{13} \mathrm{G}$, and the characteristic age is $\sim 1700$ yr. These parameters are consistent with the initial pulsar period $15 \mathrm{~ms}$, if the pulsar real age is close to its characteristic age. However, in order to fit the observed dimensions of this nebula, $5^{\prime} \times 10^{\prime}$ (Seward et al. 1984), we have to assume that the expansion velocity of the bulk matter in this nebula is much higher than applied in the modelling of other nebulae, and equal to $5000 \mathrm{~km} \mathrm{~s}^{-1}$. The initial mass of the nebula MSH15-52 is taken as in other cases equal to $4 M_{\odot}$.

For the above initial parameters of the pulsar PSR 1509-58 and its nebula, we have calculated the expected synchrotron and IC spectrum. The consistency with the observed spectrum below $1 \mathrm{MeV}$ is obtained for the differential spectral index of leptons equal to 2.1, the efficiency of acceleration of iron

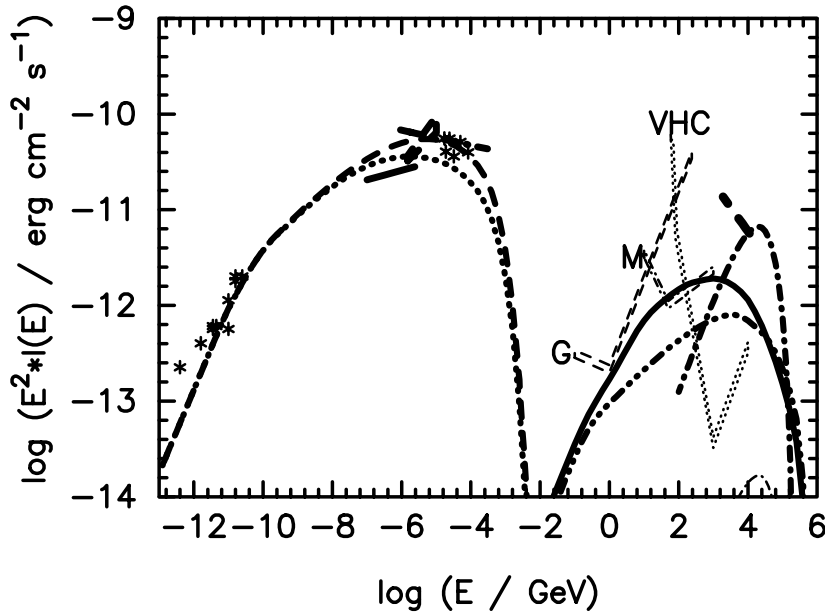

Fig. 9. The multiwavelength spectrum of the nebula MSH15-52, from the radio up to the $\mathrm{TeV} \gamma$-rays (see text for details). It is compared with the synchrotron and IC spectra produced by leptons which scatter the MBR and and synchrotron photons (dashed and dot-dot-dot-dashed curves, respectively). The synchrotron and IC spectra, calculated with the presence of additional target of infrared photons inside the nebula, are shown by the dotted and full curves, respectively. The initial parameters of the pulsar are: the period $15 \mathrm{~ms}$, the surface magnetic field $1.5 \times 10^{13} \mathrm{G}$, and the nebula are: the mass $4 M_{\odot}$, and initial expansion velocity $5000 \mathrm{~km} \mathrm{~s}^{-1}$. The real age of the object is taken to be $1700 \mathrm{yrs}$. The $\gamma$-ray spectra from decay of $\pi^{\circ}$, produced by nuclei, are shown in the case of a supernova in the typical galactic medium with density of $0.3 \mathrm{~cm}^{-3}$ (thin dot-dashed curve), and in the high density medium with the average density of $300 \mathrm{~cm}^{-3}$ (thick dot-dashed curve). The 1 year sensitivity of the planned $\gamma$-ray satellite experiment is marked by the double dashed curve ( $\mathrm{G}$ - GLAST ), and the $5 \sigma$ sensitivities (during 50 hours, $>10$ photons) of the ground Cherenkov experiments are marked by the double dot-dashed curve (M - MAGIC), and double dotted curve (VHC - VERITAS, HESS, and CANGAROO III).

nuclei $\chi=0.85$, and the conversion efficiency of energy from nuclei to leptons equal to $\xi=0.4$ (see thick dashed curve in Fig. 9). However, the IC spectrum is about an order of magnitude below the TeV $\gamma$-ray flux (Sako et al. 2000, see thick dashed line in Fig. 9). This low $\gamma$-ray flux is caused by large adiabatic energy losses due to the fast expansion of the nebula. Even if we assume the existence of additional target of thermal infrared photons in the nebula (see e.g. Arendt 1989), with the temperature $100 \mathrm{~K}$ (as in the case of the Crab), and the energy density five times higher than the energy density of MBR, the IC spectrum can not explain the CANGAROO observations (see thin full curve in Fig. 9). The $\gamma$-ray emission from decay of $\pi^{\mathrm{o}}$, produced in the interactions of nuclei accumulated inside this nebula (thick dot-dashed curve in Fig. 9), is much below the IC spectrum produced by leptons.

However there are evidences of a dense matter close to this supernova remnant. The thermal, optical nebula, RCW 89, containing $\mathrm{H}_{\alpha}$ line-emitting filaments with density $\sim 5 \times 10^{3} \mathrm{~cm}^{-3}$, coincides with the NW component of the remnant MSH15-52 (Seward et al. 1983). If the nuclei injected by the pulsar are captured by this high density filaments, then the reported level of $\mathrm{TeV} \gamma$-ray emission from this object might be due to the $\gamma$-rays produced in the interactions of nuclei with the matter 
with the average density of $\sim 300 \mathrm{~cm}^{-3}$ (see thin dot-dashed curve in Fig. 9). However, even in the most pessimistic case, the IC spectra produced by leptons in MSH15-52 should be detected by the new generation of Cherenkov telescopes at $\sim 1 \mathrm{TeV}$ (see the sensitivity limit of the CANGAROO III and HESS telescopes marked by the double dotted curves in Fig. 9).

\subsection{The Nebula 3C58 around PSR J0205+6449}

The main features of the supernova remnant, 3C58, with its pulsar PSR J0205+6449, are quite similar to the Crab pulsar and Nebula. In fact, the PSR J0205+6449 period of $65 \mathrm{~ms}$, and estimated surface magnetic field of $3.6 \times 10^{12} \mathrm{G}$, the distance of $3.2 \mathrm{kpc}$ (Roberts et al. 1993), and the size of the nebula (a factor of two larger than the Crab Nebula) makes this object the closest to the Crab pulsar and Nebula. Therefore, 3C58 should be considered as a good candidate for the $\mathrm{TeV} \gamma$-ray source. Moreover, the pulsar PSR J0205+6449 and its nebula 3C58 are probably very young, sometimes identified with the historic supernova in $1181 \mathrm{yr}$ (Thorsett et al. 1992). The multiwavelength spectrum of this nebula extends from the radio (Green 1986) up to the X-rays (ASCA - Torii et al. 2000). The spectrum is additionally constrained by the upper limits in the infrared (Green $\&$ Scheuer 1992), and the $\gamma$-rays by the EGRET (Fichtel et al. 1994) and the Whipple (Hall et al. 2001). It is on a much lower level than observed from the Crab Nebula (see Figs. 6 and 10 for comparison).

Let us model the low energy spectrum of this nebula assuming that its real age is $\sim 820$ yrs, i.e. consistent with the age of the SN $1181 \mathrm{yr}$. Then, the required initial period of the pulsar has to be $\sim 60 \mathrm{~ms}$ and the expansion velocity of the nebula $\sim 5000 \mathrm{~km} \mathrm{~s}^{-1}$, in order to fit the observed parameters of the pulsar and the dimension of the nebula. We calculate the synchrotron spectrum from this nebula applying other parameters of the model consistent with that ones derived for the Crab Nebula, i.e. the lepton spectrum, $\chi=0.85$, and $\xi=0.5$. However, since the shape of calculated spectrum (see dotted curve in Fig. 10) is completely different than the observed one, it is rather unlikely that this PWNa can be described properly by the above set of parameters.

Therefore, we followed the proposition by Bietenholz et al. (2001), who argue that the real age of 3C58 is $\sim 5000$ yrs, consistent with the characteristic age of the pulsar PSR J0205+6449 (Murray et al. 2002). For this age, the initial period of the pulsar has to be $15 \mathrm{~ms}$, and the expansion velocity $1000 \mathrm{~km} \mathrm{~s}^{-1}$, as proposed by Fesen (1983). We have calculated the synchrotron spectrum from 3C58, applying the above initial parameters. The obtained fit to the radio$\mathrm{X}$-ray spectrum, assuming $\chi=0.85$ and $\xi=0.07$, is reasonable (see dashed curve in Fig. 10). Note that, much lower value for the efficiency of lepton acceleration, in respect to the Crab Nebula, is required in order to be consistent with the low X-ray flux from this nebula. The calculated $\gamma$-ray emission from ICS (full curve) and from hadronic interactions (dot-dashed curve) is about $\sim 10^{-13} \mathrm{erg} \mathrm{cm}^{-2} \mathrm{~s}^{-1}$. This is significantly below the $5 \sigma$ sensitivity limit of the MAGIC telescope during the 50 hour observation (double dot-dashed line in Fig. 10). However it is

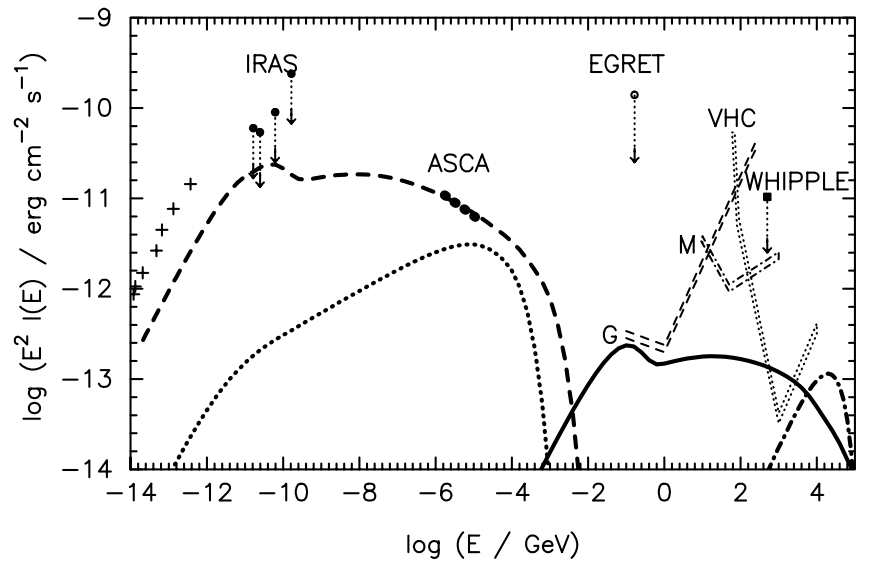

Fig. 10. The multiwavelength spectrum of the nebula 3C58 (see text for details) is compared with the synchrotron spectrum (dashed curve) and the IC spectrum (full curve) produced by leptons inside the nebula. The initial parameters of the pulsar and the supernova are as in the case of the Crab Nebula, accept the surface magnetic field $3.6 \times 10^{12} \mathrm{G}$, and the age of 5000 yrs. The $\gamma$-ray spectrum from decay of pions is shown by the dot-dashed curves. The dotted curve shows the synchrotron spectrum from the nebula assuming that this object is connected with the supernova in $1181 \mathrm{yr}$, which require the initial period of the pulsar $60 \mathrm{~ms}$, and the expansion velocity of the nebula $5000 \mathrm{~km} \mathrm{~s}^{-1}$. The sensitivities of the planned experiments are shown as in Fig. 9.

close to the $5 \sigma$ sensitivity limit of the VERITAS telescopes during 50 hour observation (see double dotted broken line in Fig. 10). Therefore, we conclude that predicted $\gamma$-ray emission above $>100 \mathrm{GeV}$ from the nebula 3C 58, can be detected by the VERITAS telescopes if this source is observed longer than 50 hours.

\subsection{The Nebula CTB 80 around PSR $1951+32$}

The pulsar PSR 1951+32 has a short period, $39.5 \mathrm{~ms}$, large characteristic age of $1.1 \times 10^{5} \mathrm{yr}$, and a relatively weak surface magnetic field $4.9 \times 10^{11} \mathrm{G}$. It is inside the supernova remnant CTB 80, which consists of a $10^{\prime} \times 6^{\prime}$ compact radio nebula immersed in a radio shell-like extended source with a diameter of 30' (e.g. Velusamy et al. 1976; Angerhofer et al. 1981). The differential radio spectrum has the average spectral index of -0.6 . The estimated dynamic age of the shell-like nebula $9.6 \times 10^{4} \mathrm{yr}$ (for the distance $2.5 \mathrm{kpc}$ ) agrees well with the characteristic age of the pulsar (Koo et al. 1990). In the X-rays, the nebula is composed of a compact nebula of $\sim 1^{\prime}$ and the diffuse nebula with the radius of $\sim 5^{\prime}$ (Safi-Harb et al. 1995). The differential X-ray spectrum of the compact nebula is equal to 2.1 and luminosity $3.9 \times 10^{33} \mathrm{ergs} \mathrm{s}^{-1}$. When modelling this nebula, we consider two possibilities.

In the first one, we assume that these objects are at the distance of $2.5 \mathrm{kpc}$ and have the real age close to the pulsar's characteristic age. Then, the observed parameters of the pulsar and nebula are consistent with the initial period of the pulsar of $15 \mathrm{~ms}$ and the initial expansion velocity of the shell-like nebula equal to $2000 \mathrm{~km} \mathrm{~s}^{-1}$, as in the case of the Crab Nebula. The synchrotron spectrum calculated for these parameters fits quite well to the observations from the radio up to the X-rays 


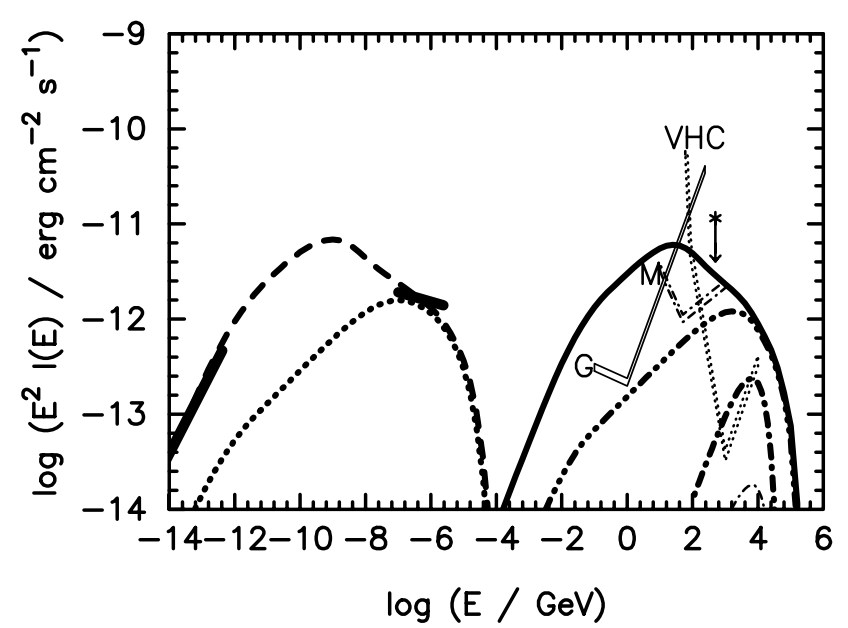

Fig. 11. The multiwavelength spectrum of the nebula CTB 80, around PSR 1951+32, (see text for details) is compared with the synchrotron spectrum (thick dashed curve) produced by leptons in the inner compact nebula, and the IC spectrum produced by leptons which comptonize the MBR in the compact nebula (full curve). The initial parameters of the pulsar and the supernova are as in the case of the Crab Nebula, accept the acceleration efficiency of leptons, $\xi=0.15$ and their spectral index equal to 2.3. The surface magnetic field of the pulsar is equal to $4.9 \times 10^{11} \mathrm{G}$, and the real age is $10^{5} \mathrm{yrs}$. The $\gamma$-ray spectrum from decay of pions produced by nuclei within the shelllike radio nebula is shown by the thin dot-dashed curve. If the nebula is the remnant of the supernova at the year 1408, then the expected synchrotron and IC spectra from leptons are shown by the dotted and dot-dot-dot-dashed curves and the spectrum from decay of pions by the thick dot-dashed curve.

(see thick dashed curve in Fig. 11). However, in order to obtain better fit we slightly modified the parameters derived for the Crab Nebula, by changing the efficiency of positron acceleration to $\xi=0.15$, and the spectral index of injected positrons to 2.3. The IC spectrum produced by leptons inside the compact nebula is shown by the thick full curve in Fig. 11 . This $\gamma$-ray emission should be easily detected by the GLAST detector in the GeV energies and by the Cherenkov telescopes (MAGIC, VERITAS) at $\mathrm{TeV}$ energies. The $\gamma$-ray emission from decay of pions, produced in collisions of nuclei, is negligible for the nebula with the age $\sim 10^{5}$ yrs (see thick dot-dashed curve in Fig. 11).

In the second modelling, we follow the claims that the nebula CTB 80 is the remnant of the recent supernova at the year 1408, found in the Chinese recorders (Strom et al. 1980; Wang \& Seward 1984). Then, the initial period of the pulsar has to be very close to the observed one, i.e. equal to $\sim 39.35 \mathrm{~ms}$, due to the low surface magnetic field. In this case, the radio shell-like extended nebula with a diameter of $30^{\prime}$ can not be related to the CTB 80 at all. To fit the observed size of the compact nebula, the expansion velocity of the bulk matter of this supernova has to be $\sim 1000 \mathrm{~km} \mathrm{~s}^{-1}$. We have tried to describe the observed X-ray spectrum of the compact nebula around PSR $1951+32$, assuming that the mass of the nebula is also equal to $4 M_{\odot}, \chi=0.85, \xi=0.2$, and the index of the power law spectrum of injected leptons equal as before to 2.3. The corresponding synchrotron and IC spectra from leptons are shown in Fig. 11 by the thin dashed and full curves. Although, we are able to fit reasonably well the observed X-ray spectrum, the lower part of synchrotron spectrum is significantly below the level of the radio emission. However, since the radio emission corresponds to a much larger region than the X-ray compact nebula, connected rather with the extended nebula with the radius of a $10^{\prime} \times 6^{\prime}$, we can not reject the connection of the CTB 80 with the supernova at the year 1408 . We have also calculated the $\gamma$-ray emission from decay of pions assuming the above parameters of the nebula. These emission can slightly contribute to the IC spectrum at $\sim 10 \mathrm{TeV}$.

\section{Conclusion}

We have applied the model for the acceleration of positrons in resonant interactions with the heavy nuclei (see e.g. Arons 1998) to construct a time dependent model for the radiation processes in the PWNe. We calculate self-consistently the amount of relativistic nuclei and leptons inside the PWNa as a function of time, measured from the supernova explosion, i.e. formation of a pulsar. The model describes well the lower energy part of the broad band spectrum observed from the Crab Nebula by the synchrotron spectrum of leptons. The $\gamma$-ray spectrum below a few $\mathrm{TeV}$ is described by the inverse Compton spectrum from scattering of low energy synchrotron photons, microwave background radiation, and infrared photons by leptons, and above $\sim 10 \mathrm{TeV}$ by the $\gamma$-rays from decay of pions produced in hadronic collisions of nuclei. From the fitting to the Crab Nebula spectrum, we derive the free parameters of this model. These parameters are used as frequently as possible in modelling of other nebulae from which the $\mathrm{TeV} \gamma$-ray emission have been reported. We consider two southern sky nebulae, around the Vela pulsar (89 ms period) and around PSR 1706-44 (102 ms period), which have quite similar parameters. By using these same initial periods of the pulsars and the expansion velocities of their nebulae, as obtained from modelling of the Crab pulsar, we get the observed values for these two pulsar's periods and dimensions of their nebulae. We have obtained reasonable fitting to the $\mathrm{X}$-ray and $\mathrm{TeV} \gamma$-ray flux from the Vela nebula for the model of acceleration of leptons as defined in the case of the Crab Nebula. However, the X-ray luminosity of the nebula around PSR 1706-44 is significantly lower than that observed from the Crab Nebula. Therefore, we have to apply much lower efficiency of acceleration of leptons in this nebula in order to explain the X-ray spectrum by synchrotron process. In such a case the calculated $\mathrm{TeV} \gamma$-ray flux from IC scattering of the MBR by leptons is a few times below the fluxes reported by the CANGAROO and the Durham groups (Kifune et al. 1995; Chadwick et al. 1998). These observations can be explained by this model if we postulate the existence of additional photon target for leptons inside the nebula, e.g. thermal infrared photons.

Next, we interpret the southern sky nebula MSH15-52 (containing the $\gamma$-ray pulsar PSR 1509-58), which has been marginally detected at the TeV energies by the CANGAROO telescope (Sako et al. 2000). The observed period of PSR 1509-58 is consistent with the initial period of $15 \mathrm{~ms}$, 
as in the case of the Crab pulsar. But, the dimensions and the age of the nebula argue for a much higher expansion velocity of this nebula. We obtained reasonable fit to the radio and X-ray emission from MSH15-52. However, calculated selfconsistently TeV $\gamma$-ray flux is about an order of magnitude below that one reported by Sako et al. (2000). This nebula is close to the dense optical nebula RCW 89. If significant amount of nuclei injected by the pulsar is captured by these high density region, then the $\gamma$-rays from decay of pions, produced in hadronic collisions, can be explain the level of reported $\mathrm{TeV}$ emission. Therefore, we suggest that the $\mathrm{TeV}$ emission reported by the CANGAROO can have hadronic origin in contrary to the calculations of Du Plessis et al. (1995), who argue for the dominance of leptonic emission also in this energy range.

Based on the experience reached from modelling of the objects for which $\mathrm{TeV} \gamma$-ray emission has been reported, we try to predict the level of the high energy $\gamma$-ray emission from other nebulae, which may become good potential targets for the next generation of the GeV-TeV $\gamma$-ray telescopes. We concentrate on the two northern sky nebulae: $3 \mathrm{C} 58$ and CTB 80, which contain fast pulsars PSR J0205+6449 (65 ms) and PSR 1951+32 (39.5 ms), respectively. It is found that 3C58 is too weak to be detected in the GeV energy range by the GLAST detector and lays on the sensitivity limit of the VERITAS telescope in the $\mathrm{TeV}$ energies. From another site, CTB 80 should be easily detected by the GLAST detector and the MAGIC and VERITAS telescopes.

The experience reached from these modelling convince us that it is rather difficult to describe consistently the high energy processes in the PWNe by a simple model with a small number of parameters. It looks that individual sources require different approach which is determined by the specific conditions around and inside the nebula, like for example the presence of a dense matter or additional targets of soft photons appart from the synchrotron radiation produced inside the nebula and MBR.

Our modelling base on the assumption that most of the rotational energy of the pulsars are injected in the form of relativistic nuclei. Heavy nuclei, which escape from the PWNe, can contribute significantly to the cosmic ray spectrum at energies above the knee region (Bednarek \& Protheroe 2002), at which the enhancement of heavy composition in the cosmic ray spectrum is reported by some experiments. Another independent test on the importance of injection of nuclei by the pulsars and on the validity of the considered here model for the acceleration of leptons can be provided by the observations of the high energy neutrinos from the PWNe by the future neutrino telescopes (IceCube, ANTARES, NESTOR). We have considered this problem in an accompanying paper (Bednarek 2003). It is shown there that, in contrary to previous estimates (Guetta \& Amato 2003), only very young nebulae (of the Crab type) can produce observable neutrino signals in a $1 \mathrm{~km}^{2}$ detector.

Acknowledgements. This work is supported by the Polish KBN grants No. 5P03D 02521 and PBZ-KBN-054/P03/2001.

\section{References}

Abrahams, A. L., \& Shapiro, S. L. 1991, ApJ, 374, 652
Aharonian, F. A., \& Atoyan, A. M. 1995, Astropart. Phys., 3, 275

Aharonian, F., Atoyan, A. M., \& Kifune, T. 1997, MNRAS, 291, 162

Angerhofer, P. E., Strom, R. G., Velusamy, T., \& Kundu, M. R. 1981, A\&A, 94, 313

Arons, J. 1998, Proc. Elba Conf. on Pulsars and their Nebulae, Mem. Soc. Ast. Ital., v. 69, p. 989

Atoyan, A. M., \& Aharonian, F. A. 1996, MNRAS, 278, 525

Becker, W., Brazier, K. T. S., \& Trümper, J. 1995, A\&A, 298, 528

Bednarek, W. 2003, A\&A, in press

Bednarek, W., \& Protheroe, R. J. 1997, PRL, 79, 2616

Bednarek, W., \& Protheroe, R. J. 2002, Astropart. Phys., 16, 397

Caraveo, P. A., De Luca, A., Mignani, R. P., \& Bignami, G. F. 2001, ApJ, 561, 930

Bietenholz, M. F., Kassim, N. E., \& Weiler, K. W. 2001, ApJ, 560, 772

Cha, A., Sembach, K. M., \& Danks, A. C. 1999, ApJ, 515, L25

Chadwick, P. M., Dickinson, M. R., Dipper, N. A., et al. 1998, Astropart. Phys., 9, 131

Chadwick, P. M., Lyons, K., McComb, T. J. L., et al. 2000, ApJ, 537, 414

Cheng, K. S., Cheung, T., Lau, M. M., Yu, K. N., \& Kwok, W. M. 1990, J. Phys. G, 16, 1115

Cheng, K. S., Ho, C., \& Ruderman, M. 1986, ApJ, 300, 500; ApJ, 300, 525

Chevalier, R. A. 2003, Adv. Space Res., in press [astro-ph/0301370]

Davidson, K., \& Fesen, R. A. 1985, ARA\&A, 23, 119

De Jager, O. C., \& Harding, A. K. 1992, ApJ, 396, 161

De Jager, O. C., Harding, A. K., Michelson, P. F., et al. 1996, ApJ, 457,253

De Jager, O. C., Harding, A. K., \& Strickman, M. S. 1996, ApJ, 460, 729

Dodson, R., \& Golap, K. 2002, MNRAS, 334, 307

Du Plessis, De Jager, O. C., Buchner, S., et al. 1995, ApJ, 453, 746

Fegan, S. 2001, in Proc. The nature of unidentified galactic high energy gamma ray sources, ed. A. Carraminana et al., Tonantzintla, Mexico, Astrophys. \& Space Science Library, 267, 285

Fesen, R. A. 1983, ApJ, 270, L53

Finley, J. P., Srinivasan, R., Saito, Y., Hiriyama, M., \& Kamae, T. 1998, ApJ, 493, 884

Fichtel, C. E. Bertsch, D. L., Chiang, J., et al. 1994, APJS, 94, 551

Frail, D. A., Goss, W. M., \& Whiteoak, J. B. Z. 1994, ApJ, 437, 781

Gallant, Y. A., \& Arons, J. 1994, ApJ, 435, 230

Gallant, Y. A. E., van der Swaluw, J. G., Kirk, \& Achterberg, A. 2002, in Proc. Neutron stars in Supernova Remnants, ed. P. O. Slane, \& B. M. Gaensler, ASP Conf. Ser., 271, 99

Gaensler, B. M., Brazier, K. T. S., Manchester, R. N., Johnston, S., \& Green, A. J. 1999, MNRAS, 305, 724

Gould, R. J. 1965, PRL, 15, 577

Goldreich, P., \& Julian, W. H. 1969, ApJ, 157, 869

Green, D. A. 1986, MNRAS, 218, 533

Green, D. A., \& Schreuer, P. A. G. 1992, MNRAS, 258, 833

Grindlay, J. E., \& Hoffman, J. A. 1971, Astrophys. Lett., 8, 209

Guetta, D., \& Amato, E. 2003, Astropart. Phys., 19, 403

Harnden, F. R., Grant, P. D., Seward, F. D., \& Kahn, S. M. 1985, ApJ, 299, 828

Hillas, A. M., Akerlof, C. W., Biller, S. D., et al. 1998, ApJ, 503, 744

Hall, T. A., Wakely, S. P., et al. 2001, Proc. 27th ICRC (Hamburg) (Copernicus Gesellschaft 2001), p. 2485

Hoshino, M., Arons, J., Gallant, Y. A., \& Langdon, B. 1992, ApJ, 390, 454

Kanbach, G., Arzoumanian, Z., Bertsch, D. L., et al. 1994, A\&A, 289, 855

Kifune, T., Tanimori, T., Ogio, S., et al. 1995, ApJ, 438, L91 
Kennel, C. F., \& Coroniti, F. V. 1984, ApJ, 283, 710

Koo, B., Reach, W. T., Heiles, C., Fesen, R. A., \& Shull, J. M. 1990, ApJ, 346, 178

Levine, S. H., Lang, F. L., Lewin, W. H. G., et al. 1984, ApJS, 54, 581

Longair, M. S. 1981, High Energy Astrophysics (Cambridge University Press), 279

Marsden, D., Blanco, P. R., Gruber, D. E., et al. 1997, ApJ, 491, L39

McAdam, W. B., Osborne, J. L., \& Parkinson, M. L. 1993, Nature, 361,516

Mineo, T., Cusumano, G., Maccarone, M. C., Massaglia, S., et al. 2001, A\&A, 380, 695

Murray, S. S., Slane, P. O., Seward, F. D., Ranson, S. M., \& Gaensler, B. M. 2002, ApJ, 568, 226

Nomoto, K., \& Tsuruta, S. 1987, ApJ, 312, 711

Ostriker, J. P., \& Gunn, J. E. 1971, ApJ, 164, L95

Ögelmanan, H., Finley, J. P., \& Zimmermann, H.-U. 1993, Nature, 361,136

Ögelman, H., \& Zimmermann, H.-U. 1989, A\&A, 214, 179

Pacini, F., \& Salvati, M. 1973, ApJ, 186, 249

Pravdo, S. H., Becker, R. H., Boldt, E. A., et al. 1978, ApJ, 223, 537

Rees, M. J., \& Gunn, J. E. 1974, MNRAS, 167, 1

Reynolds, S. P., \& Chevalier, R. A. 1984, ApJ, 278, 630

Rieke, G. H., \& Weekes, T. C. 1969, ApJ, 155, 429

Sako, T., Matsubara, Y., Muraki, Y., Ramanamurthy, P. V., Dazeley, S. A., et al. 2000, ApJ, 537, 422
Roberts, D. A., Goss, W. M., Kalberla, P. M. W., Herbstmeier, U., \& Schwarz, U. J. 1993, A\&A, 274, 427

Safi-Harb, S., Ögelman, H., \& Finley, J. P. 1995, ApJ, 439, 722

Seward, F. D., Harnden, F. R. Jr., Murdin, P., \& Clark, D. H. 1983, ApJ, 267, 698

Seward, F. D., Harnden, F. R. Jr., Szymkowiak, A., \& Swank, J. 1984, ApJ, 281, 650

Stepanian, A. A. 1980, Izv. Krymskoi Astrofiz. Obs., 62, 79

Strom, R. G., Angerhofer, P. E., \& Velusamy, T. 1980, Nature, 284, 38

Taylor, J. H., \& Cordes, J. M. 1993, ApJ, 411, 674

Thorsett, S. E. 1992, Nature, 356, 690

Torii, K., Slane, P. O., Kinugasa, K., Hashimotodani, K., \& Tsunemi, H. 2000, PASJ, 52, 875T

Trussoni, E., Massalia, S., Caucino, S., Brinkmann, W., \& Aschenbach, B. 1996, A\&A, 306, 581

Usov, V. V., \& Melrose, D. B. 1995, Austr. J. Phys., 48, 571

Wang, Z. R., \& Seward, F. D. 1984, ApJ, 285, 607

Wdowczyk, J., \& Wolfendale, A. W. 1987, J. Phys. G, 13, 411

Weekes, T. C., Cawley, M. F., Fegan, S., et al. 1989, ApJ, 342, 379

Willmore, A. P., Eyles, C. J., Skinner, G. K., \& Watt, M. P. 1992, MNRAS, 254, 139

Velusamy, T., Kundu, M. R., \& Becker, R. H. 1976, A\&A, 51, 21

Yoshikoshi, T., Kifune, T., Dazeley, S. A., et al. 1997, ApJ, 487, L65 